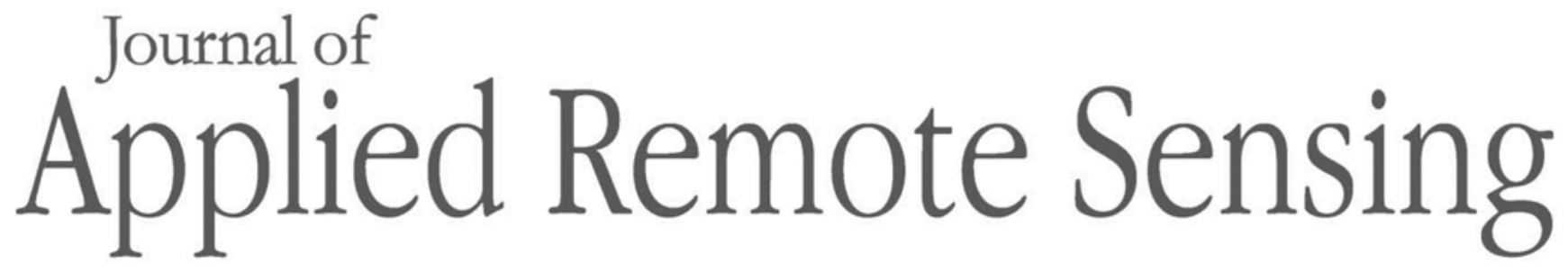

RemoteSensing.SPIEDigitalLibrary.org

\title{
Vicarious calibration correction of large FOV sensor using BRDF model based on UAV angular spectrum measurements
}

\author{
Zhiqiang Pan \\ Haoping Zhang \\ Xiangjun Min \\ Zhaopeng $\mathrm{Xu}$
}




\title{
Vicarious calibration correction of large FOV sensor using BRDF model based on UAV angular spectrum measurements
}

\author{
Zhiqiang Pan,* Haoping Zhang, Xiangjun Min, and Zhaopeng Xu \\ China Centre for Resources Satellite Data and Application, Beijing, China
}

\begin{abstract}
When a satellite sensor with a large field of view and wide swath is calibrated, it is not easy to obtain the image when the calibration site is located precisely at the nadir position. If the location of a calibration site is at an off-nadir position in the image, calibration errors will be caused by the inconsistent observation angle between the sensor view and the ground measurement view. The bidirectional reflectance distribution function (BRDF) model plays an important role in solving this problem. In this study, a BRDF measurement system based on an unmanned aerial vehicle (UAV) is developed. This system has the capability of measuring angular data with observation azimuth angle ranging from $0 \mathrm{deg}$ to $360 \mathrm{deg}$ with an angle interval of $30 \mathrm{deg}$, and observation zenith angle ranging from 0 deg to $50 \mathrm{deg}$ with an angle interval of $10 \mathrm{deg}$. The directional data of the Dunhuang calibration site were measured using the UAV BRDF measuring system at different solar zenith and azimuth angles, and the spatiotemporal distribution characteristic of forward- and backward-scattering of Dunhuang calibration site was analyzed. A Ross-Li BRDF model, built using measurement data, is used to calculate the directional surface reflectance under any observation geometry of solar and satellite. These calculations are applied to correct the calibration data of the CBERS-04 WFI sensor. Results show that the BRDF model significantly improves the calibration accuracy, especially in the case of large observation angles. () The Authors. Published by SPIE under a Creative Commons Attribution 4.0 Unported License. Distribution or reproduction of this work in whole or in part requires full attribution of the original publication, including its DOI. [DOI: 10.1117/1.JRS.14.027501]
\end{abstract}

Keywords: radiometric calibration; large FOV sensor; BRDF model; UAV.

Paper 190866 received Nov. 3, 2019; accepted for publication Mar. 17, 2020; published online Apr. 2, 2020.

\section{Introduction}

Absolute radiometric calibration of satellite sensors is required for the quantitative application of satellite data. Vicarious calibration is an important method for the calibration of satellite sensors. Typically, this is done using the reflectance-based calibration method, in which two kinds of parameters are measured-field surface reflectance and atmospheric parameters (such as aerosol levels, temperature, and humidity profile)—while the satellite is passing over the calibration site. All the parameters together with the observed geometric parameters of solar and the satellite sensor are input into the radiative transfer model (6S or MODTRAN) to obtain the top-ofatmosphere (TOA) radiance of the satellite sensors, and the calibration coefficient is calculated according to the TOA radiance and digital number (DN) value of the calibration site in the satellite image. ${ }^{1,2}$

During the vicarious calibration process, surface reflectance is the most important parameter since the measurement error of surface reflectance can be completely transferred to the calibration result (calibration coefficient). For the calibration of a satellite sensor with a large field of view (FOV), the surface reflectance error can be caused by the off-nadir observation angle of the calibration site. In general, the natural landscape is not Lambertian, instead exhibiting anisotropic characteristics even if the calibration site is homogeneous, meaning that the surface reflectance is different at different viewing angles of the calibration site. When the sensor is calibrated, the surface reflectance of the calibration site is manually measured vertically downward (nadir)

*Address all correspondence to Zhiqiang Pan, E-mail: panzhiqiang2011@126.com 
and is used to calculate the sensor reflectance. Usually, however, the large FOV sensor observes the calibration site at the off-nadir angle. ${ }^{3}$ The difference in viewing angle between satellite and measurement data can lead to surface reflectance errors. To ensure the precision of calibration results, the observation angle difference should be considered by correcting the viewing angle of the measurement data to the viewing angle of the sensor. The bidirectional reflectance distribution function (BRDF) model, which can describe the anisotropic reflectance characteristic of a surface, plays an important role in solving this problem.

Based on their various construction mechanisms, BRDF models can be divided into empirical statistical models, physical models, and semiempirical models. The semiempirical model, also known as the kernel-driven model, was first proposed by Roujean et al. ${ }^{4}$ It combines a linear volume-scattering radiative transfer model and a surface-scattering geometric model and soon became the most widely used model in BRDF research and application. ${ }^{5}$ For the kerneldriven model, the Ross-Li model, which is composed of a volumetric scattering kernel (RossThick kernel) and a geometric optical kernel (LiSparse kernel), ${ }^{6}$ is more commonly used. To establish an operational Ross-Li BRDF model, multiangle observation data for the target are needed. The three weight parameters of the model representing the isotropic, geometric, and volumetric kernels can then be calculated by the least squares method using observation data, ultimately allowing the model to be used for simulating the bidirectional reflectance at any desired illumination and viewing angle. The multiangle observation data used for establishing the Ross-Li BRDF model can be either the satellite multiangle observation data or the angular measurement data using a goniometer instrument in the field.

The BRDF model can be established using satellite multiangle observation data. The Moderate Resolution Imaging Spectroradiometer (MODIS) BRDF product has made use of a semiempirical kernel-driven bidirectional reflectance model along with multidate, multispectral data at different observation angles to provide model parameters describing the BRDF of the land surface every 16 days since $2000 .^{7-9}$ Some researchers perform calibration studies using MODIS BRDF products. Patel et al. ${ }^{10}$ implemented the post-launch vicarious calibration of an Indian meteorological satellite by considering the effects of surface anisotropy on TOA radiance using MODIS BRDF products. Feng et al. ${ }^{11}$ studied the cross calibration between the wide field viewer sensor of the GF-1 satellite and the MODIS, for which the bidirectional effects were corrected using MODIS BRDF products. Some researchers have constructed the BRDF model using long time series and large FOV satellite images for calibration analysis. Bhatt et al. ${ }^{12}$ proposed a vicarious technique for calibrating GEO visible sensors using a kernel-based BRDF model derived over an invariant Libya-4 desert site. The Libya-4 BRDF model is TOA-based and is derived from 10 years of clear-sky Aqua satellite observations over this site. Kim et al. ${ }^{13}$ studied the anisotropy of a Sonoran desert site by widely applying previously used BRDF models to the TOA reflectance of Landsat $5 \mathrm{TM}$ data, for which the radiometric stability of the time series was computed using the BRDF-normalized TOA reflectance of the Landsat data.

Angular data measured in the field are also widely used to establish the BRDF model. Many researchers have developed different field goniometers to measure multiangular data in order to investigate BRDF effects. Some goniometers have been developed based on the principle of the field goniometer system (FIGOS) ${ }^{14}$ such as the Sandmeier field goniometer ${ }^{15}$ and the dual-view FIGOS. ${ }^{16}$ Other goniometers have been developed based on the principle of the automated spectro-goniometer, ${ }^{17}$ such as the IAC ETH,${ }^{18}$ the GOPHER,,${ }^{19}$ and the ManTIS. ${ }^{20}$ Some researchers have used new measurement tools, such as unmanned aerial vehicles (UAVs), to study the angular characteristics of ground objects. Burkart et al. ${ }^{3}$ measured the multiangular hyperspectral data of wheat using a rotary-wing UAV equipped with a spectrometer. Hakala et al. ${ }^{21}$ conducted directional imaging of snow using a consumer UAV.

Some researchers have established the BRDF model using angular data measured in the field to study the field calibration correction of satellite sensors. The Remote Sensing Group (RSG) of the Optical Sciences Center at the University of Arizona has performed field calibration of satellite sensors since the 1980s, including sensors from the Landsat series, Sentinel, Aqua, Suomi NPP, and Terra. Many sensors view the calibration site (Railroad Valley) at off-nadir angles, which has prompted the effort to develop a BRDF correction. Since 1998, RSG has been developing a BRDF measurement system - the bidirectional reflectance factor (BRF) camera-for the retrieval of bidirectional reflectance. This camera consists of a four-band imaging radiometer 
based on a two-dimensional CCD array and an 8-mm fisheye lens. ${ }^{22,23}$ During the field calibration campaign in 2018, directional reflectance studies using a mobile spectro-goniometer system-the University of Lethbridge Goniometer System developed by the University of Lethbridge ${ }^{24}$ - together with the BRF camera were employed to obtain the BRDF data for MODIS calibration correction, improving accuracy by $3 \%$ compared to the onboard calibration coefficient. $^{25}$

China has launched several series of land observation satellites, including the CBERS, HJ, GF, and ZY. Each year a calibration campaign is carried out at the Dunhuang calibration site for the visible and near-infrared sensors of China's land observation satellites using the reflectancebased method. The goal of the current study was to develop a method for improving the calibration accuracy of large FOV sensors, such as the China-Brazil Earth Resources Satellite Wide Field Imager (CBERS-04 WFI), which observe the calibration site at an off-nadir angle. First, under the framework of the reflectance-based method, field surface reflectance and atmospheric parameters were measured for CBERS-04 WFI sensor calibration. Next, a Ross-Li kernel-driven BRDF model for the Dunhuang calibration site was constructed using measured multiangular data to correct the field surface reflectance data from the nadir angle to the sensor-viewing angle. Before the BRDF model construction, multiangular spectrum data were collected by a multiangular data measurement system using a rotary-wing UAV equipped with a hyperspectral spectrometer at the Dunhuang calibration site. Finally, the corrected surface reflectance using the BRDF model together with the atmospheric parameters were all input into a radiative transfer model (MODTRAN) in order to obtain the calibration result. This result revealed that the calibration accuracy of the WFI sensors was improved significantly after BRDF correction.

\section{Materials and Methods}

\subsection{Bidirectional Reflectance Distribution Function Definitions}

The BRDF model, proposed by Nicodemus in the 1970s to describe the anisotropy of a target, is a theoretical concept that describes the relationship between a target's irradiance geometry and the viewing angle of the remote sensing system relative to the target. ${ }^{15}$ BRDF can accurately describe the relationship between solar radiation geometry and satellite observation geometry, which can be used for angular correction of measured reflectance data to improve calibration accuracy. The definition of BRDF is as follows:

$$
f\left(\theta_{s}, \phi_{s} ; \theta_{v}, \phi_{v} ; \lambda\right)=\frac{\mathrm{d} L_{v}\left(\theta_{s}, \phi_{s} ; \theta_{v}, \phi_{v} ; \lambda\right)}{\mathrm{d} E_{s}\left(\theta_{s}, \phi_{s} ; \lambda\right)}
$$

where $f$ is the BRDF, $L$ is the radiance, $E$ is the irradiance, $s$ and $v$ denote the incident (solar) and the reflected light (observation), respectively. Here $\theta_{s}$ and $\phi_{s}$ mark the solar zenith angle and the solar azimuth angle and $\theta_{v}$ and $\phi_{v}$ mark the observation zenith angle and the observation azimuth angle, respectively. The unit of BRDF is $[1 / \mathrm{sr}]$. The variables are shown in Fig. 1.

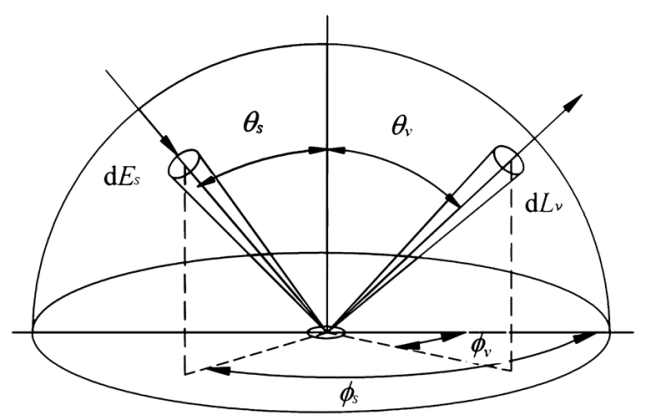

Fig. 1 Concept of incident and reflected angles in the spherical coordinate system of the BRDF. 
It is relatively difficult to measure irradiance in the field. The BRF is usually measured, which is defined as the ratio of the reflected radiance of the target to the radiance of the Lambertian reference panel under the same incident and reflected geometry conditions. The definition of $\mathrm{BRF}$ is as follows:

$$
R\left(\theta_{s}, \phi_{s} ; \theta_{v}, \phi_{v} ; \lambda\right)=\frac{L_{v}\left(\theta_{s}, \phi_{s} ; \theta_{v}, \phi_{v} ; \lambda\right)}{L_{\mathrm{ref}}\left(\theta_{s}, \phi_{s} ; \theta_{v}, \phi_{v} ; \lambda\right)}
$$

where $R\left(\theta_{s}, \varnothing_{s} ; \theta_{v}, \varnothing_{v} ; \lambda\right)$ is the $\mathrm{BRF}, L_{v}\left(\theta_{s}, \varnothing_{s} ; \theta_{v}, \varnothing_{v} ; \lambda\right)$ is the reflected radiance, and $L_{\text {ref }}\left(\theta_{s}, \varnothing_{s} ; \theta_{v}, \varnothing_{v} ; \lambda\right)$ is the radiance of the Lambertian reference panel.

For the satellite sensors, the BRF of band $i$ can be calculate by Eq. (3) using convolution between $R\left(\theta_{s}, \varnothing_{s} ; \theta_{v}, \varnothing_{v} ; \lambda\right)$ and spectral response function (SRF) of sensor band .

$$
R\left(\theta_{s}, \phi_{s} ; \theta_{v}, \phi_{v} ; \text { band }_{i}\right)=\frac{\int R\left(\theta_{s}, \phi_{s} ; \theta_{v}, \phi_{v} ; \lambda\right) \operatorname{SRF}_{\text {band }_{i}}(\lambda) \mathrm{d} \lambda}{\int \mathrm{SRF}_{\text {band }_{i}}(\lambda) \mathrm{d} \lambda},
$$

where $S R F_{\text {band }_{i}}$ is the SRF of band .

The anisotropy factor (ANIF) is also commonly used to describe the anisotropy of the target. It is defined as the normalization of reflectance data to nadir reflectance. The ANIF is used in this study to analyze the spectral variability in BRDF data.

$$
\operatorname{ANIF}\left(\theta_{s}, \phi_{s} ; \theta_{v}, \phi_{v} ; \lambda\right)=R\left(\theta_{s}, \phi_{s} ; \theta_{v}, \phi_{v} ; \lambda\right) / R_{0}\left(\theta_{s}, \phi_{s} ; \lambda\right),
$$

where $R\left(\theta_{s}, \varnothing_{s} ; \theta_{v}, \varnothing_{v} ; \lambda\right)$ is the BRF for a given different zenith and azimuth angle of solar and observation and $R\left(\theta_{s}, \varnothing_{s} ; \lambda\right)$ is the BRF of nadir viewing for different solar zenith and azimuth angles.

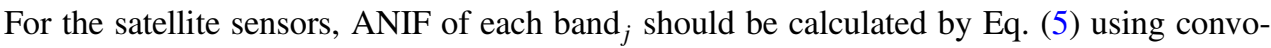
lution between $\operatorname{ANIF}\left(\theta_{s}, \varnothing_{s} ; \theta_{v}, \varnothing_{v} ; \lambda\right)$ and the $\operatorname{SRF}$ of sensor band . $_{\text {. }}$

$$
\operatorname{ANIF}\left(\theta_{s}, \phi_{s} ; \theta_{v}, \phi_{v} ; \text { band }_{j}\right)=\frac{\int \operatorname{ANIF}\left(\theta_{s}, \phi_{s} ; \theta_{v}, \phi_{v} ; \lambda\right) \operatorname{SRF}_{\text {band }_{j}}(\lambda) \mathrm{d} \lambda}{\int \operatorname{SRF}_{\text {band }_{j}}(\lambda) \mathrm{d} \lambda}
$$

where $\mathrm{SRF}_{\text {band }_{j}}$ is the SRF of band .

\subsection{Dunhuang Calibration Site}

The Dunhuang radiometric calibration site is located in the west of Dunhuang City, Gansu Province, China $\left(40.09^{\circ} \mathrm{N}, 94.39^{\circ} \mathrm{E}\right.$ ) (Fig. 2). This region features the homogeneous characteristics of a Gobi desert surface. It has been used for the calibration of China's remote sensing satellite sensors of visible, near-infrared, and shortwave infrared bands since 2000. It has an area of $40 \times 30 \mathrm{~km}^{2}$ and an altitude of $1229 \mathrm{~m}$. The most suitable time for calibration at Dunhuang is between May and October each year because the likelihood of clear days is greater and the temperatures are more favorable.

\subsection{Unmanned Aerial Vehicle Angular Measurement System}

\subsubsection{System introduction}

In this study, we developed an automatic measurement system based on an eight-rotor UAV (Fig. 3). A hyperspectral measurement instrument (SVC 1024) with the spectral range of 350 to $2500 \mathrm{~nm}$ was integrated into the UAV. The spectrum resolution of SVC 1024 is as follows: 350 to $1000 \leq 2.75 \mathrm{~nm}, 1000$ to $1900 \leq 8 \mathrm{~nm}, 1900$ to $2500 \leq 6 \mathrm{~nm}$, and the fiber-optic had an FOV of approximately $25 \mathrm{deg}$.

The UAV can fly autonomously along the planned flight route and can hover at the planned measurement points to collect data. A stabilization system was designed to stabilize the fiberoptic by adjusting pitch and roll automatically if the UAV flight attitude was changed by wind 


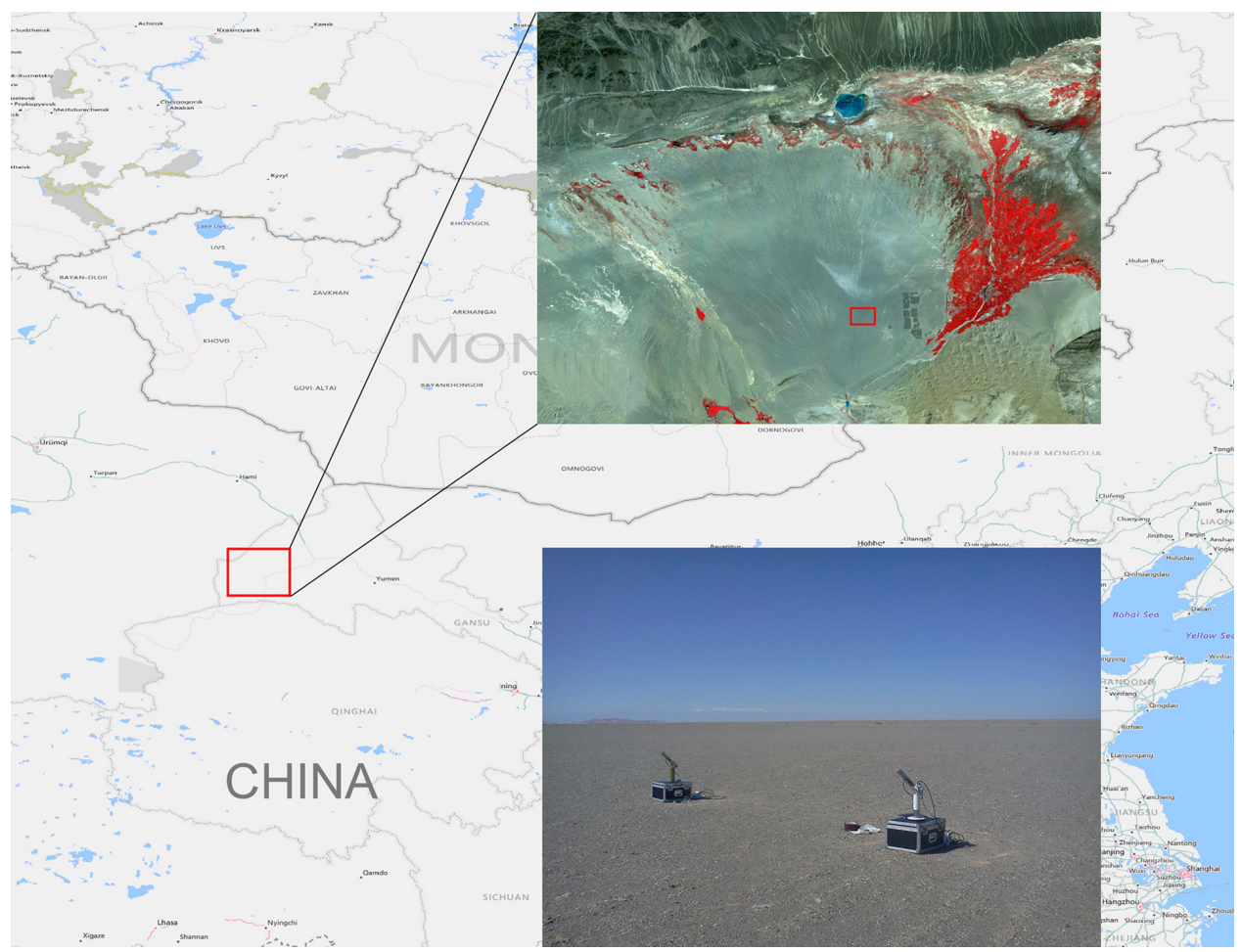

Fig. 2 Location of the Dunhuang calibration site. The red box on the satellite image is the $550 \times$ $550 \mathrm{~m}^{2}$ area in which the field calibration campaign is usually performed. The lower right image is a photograph of the natural landscape of the calibration site.
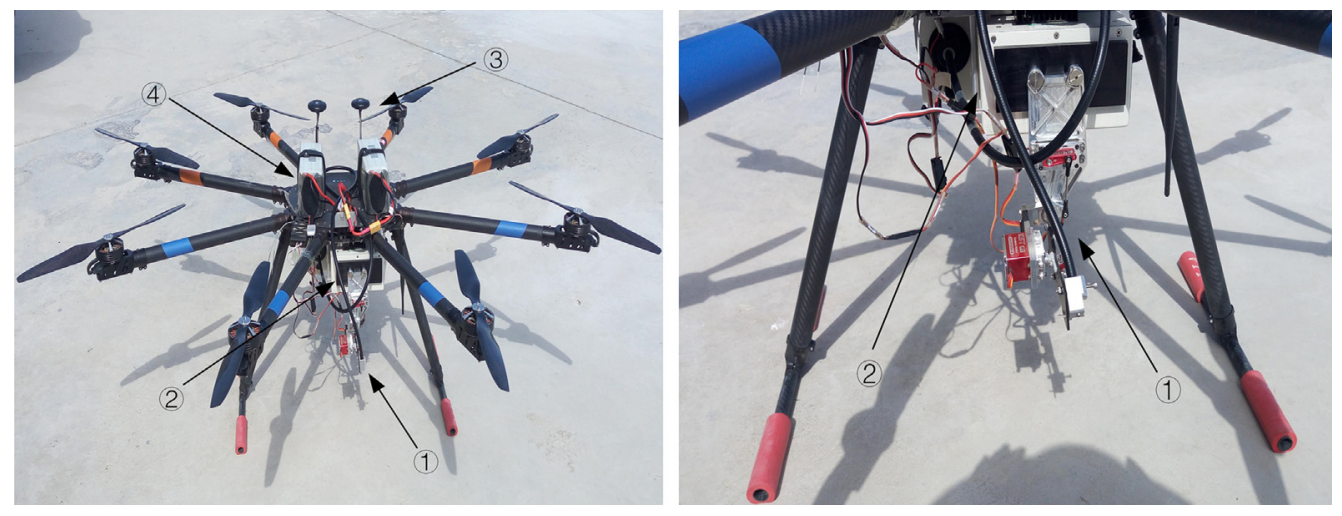

Fig. 3 Eight-rotor UAV automatic system for angular hyperspectral measurement (1) stabilization system; (2) hyperspectral measurement instrument: SVC 1024; (3) GPS; (4) battery).

during the flight. The system has the capability of angular spectrum measurement. The vertical angle (observation zenith angle) should be adjusted manually with an increment of $10 \mathrm{deg}$. For each flight, the observation zenith angle is kept in the same. The horizontal angle (observation azimuth angle) is determined by the UAV heading orientation collected using GPS receivers. Through the rational planning of flight routes and hovering points, the UAV can carry out the directional measurement of 0 deg to 360 deg observation azimuth angles and 0 deg to $50 \mathrm{deg}$ observation zenith angles. Compared to a traditional goniometer, the BRDF measurement using UAV in the field campaign has several advantages: (1) the UAV can fly higher and has larger spatial sampling area, making it capable of simulating satellite observations; (2) BRDF measurement using UAV will not damage or modify the measurement site; (3) it allows measurements over inaccessible areas such as surfaces covered by tall vegetation; and (4) it allows for cheap, fast, and effective data collection. 


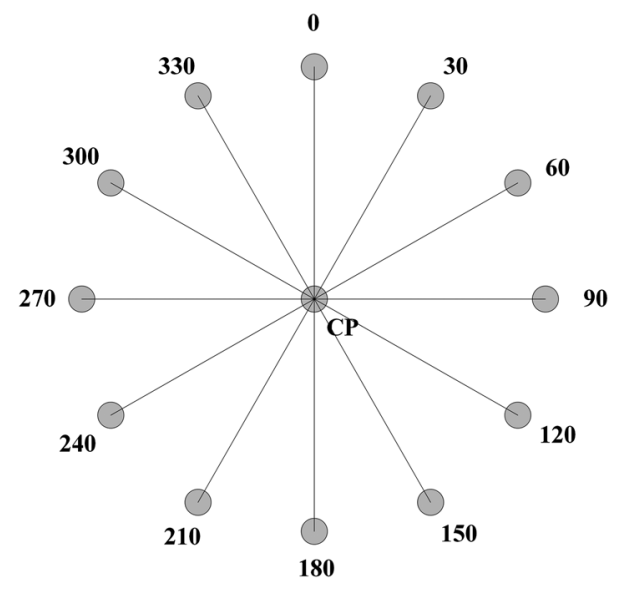

Fig. 4 Measuring points and flight route of the UAV.

\subsubsection{Flight pattern}

The BRDF observations in this study measured the center point (CP) of the hemisphere from different viewing angles. The UAV flight pattern does not fly around the CP. Rather, it flies straight through it, which allows us to determine the observation azimuth angle using UAV heading data (Fig. 4). The flight points are planned to have observation azimuth angles ranging from 0 deg to 360 deg at 30-deg intervals and observation zenith angles ranging from 0 deg to $50 \mathrm{deg}$ at 10-deg intervals. Thus, 12 points can be measured for each observation zenith angle.

The measurement process is as follows. First, we calculate the latitude and longitude of each flight point. The coordinate of the $\mathrm{CP}$ is measured in the calibration site, and the flight height is set to $20 \mathrm{~m}$, then the coordinate of each flight point can be calculated. Second, we plan the flight route according to the point coordinate and determine the flight order of the points on the route. For each observation zenith angle, the flight route is $\mathrm{CP} \rightarrow 0 \rightarrow 180 \rightarrow \mathrm{CP} \rightarrow 30 \rightarrow 210 \rightarrow \mathrm{CP} \rightarrow$ $60 \rightarrow 240 \rightarrow \mathrm{CP} \rightarrow 90 \rightarrow 270 \rightarrow \mathrm{CP} \rightarrow 120 \rightarrow 300 \rightarrow \mathrm{CP} \rightarrow 150 \rightarrow 330$ (Fig. 4), which can be used to determine the observation azimuth angle of each flight point exactly. Upon reaching a measurement point, the UAV will hover in that point for $3 \mathrm{~s}$ to measure the data.

To record the changing illumination, another spectrometer ASD on the ground is used to measure a white reference panel. Before measurement, the airborne and ground spectrometers should measure the panel simultaneously to detect the difference. The CP should be measured first in nadir view, then for each observation zenith angle at $10 \mathrm{deg}, 20 \mathrm{deg}, 30 \mathrm{deg}, 40 \mathrm{deg}$, and $50 \mathrm{deg}$. The data will be measured at 12 points along the flight route. It takes only about $2 \mathrm{~min}$ to measure 12 points. Considering the fact that UAV takeoff and landing takes time, as does manually modifying the observation zenith angle for each flight measurement, it takes about $30 \mathrm{~min}$ to complete five flights of measurements.

\subsubsection{Unmanned aerial vehicle flight position accuracy}

The accuracy of the UAV flight position is assessed by comparing the difference between the planned position and the flight position of each flight point, which is showed in Fig. 5. The planned position is the latitude and longitude coordinates of each fight point, which is calculated and uploaded in the UAV flight control system before the measurement flight. The flight position is the latitude and longitude coordinates of each fight point, which is stored in the UAV POS system during the measurement flight. As shown in Fig. 5, the deviation between planned position and the flight position is less than $1 \mathrm{~m}$, and the mean deviation is less than $0.5 \mathrm{~m}$. More importantly, almost all the flight position is located on the line between the $\mathrm{CP}$ and the planned measurement point, which can ensure the observation azimuth angle accuracy of the measurement point despite the existence of flight positioning error. It can be imagined that compared with the flight pattern of flying through CP shown in Fig. 4, if the flight pattern of flying around $\mathrm{CP}$ is adopted, the flight positioning error can directly lead to a larger observation azimuth angle error for the measurement point. 
Pan et al.: Vicarious calibration correction of large FOV sensor using BRDF model...

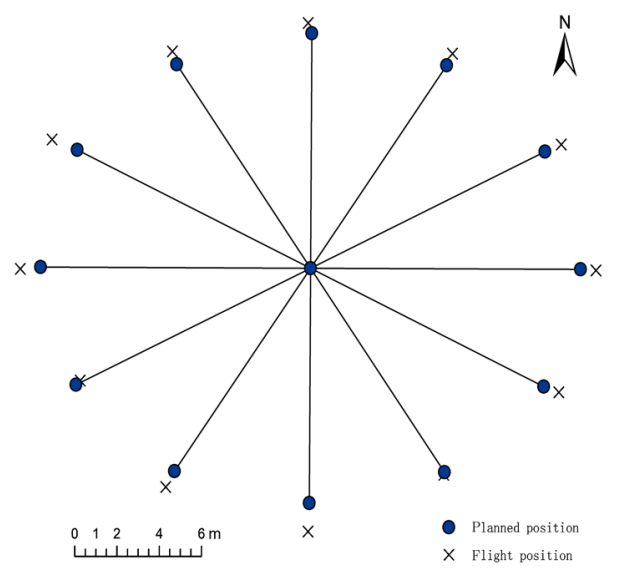

Fig. 5 The difference between the planned position and the flight position of each flight point.

\subsubsection{CBERS-04 WFI sensor}

In this study, the CBERS-04 WFI sensor was calibrated using BRDF correction. The CBERS was jointly developed by China and Brazil. It was successfully launched on December 7, 2014. The WFI, one of the payloads on the CBERS-04, has a swath of $866 \mathrm{~km}$ and a resolution of $64 \mathrm{~m}$. Table 1 lists the main parameters of the WFI sensor, and Fig. 6 shows the SRF of the CBERS-04 WFI sensor.

Table $1 \mathrm{WFI}$ sensor parameters.

\begin{tabular}{lcccccc}
\hline \hline Payload & Band & Spectrum $(\mu \mathrm{m})$ & Resolution $(\mathrm{m})$ & Swath $(\mathrm{km})$ & View angle (deg) & Revisit (day) \\
\hline \multirow{4}{*}{ WFI } & Band1 & 0.45 to 0.52 & & & & \\
& Band2 & 0.52 to 0.59 & 64 & 866 & \pm 32 & 3 \\
& Band3 & 0.63 to 0.69 & & & \\
& Band4 & 0.77 to 0.89 & & & \\
\hline \hline
\end{tabular}

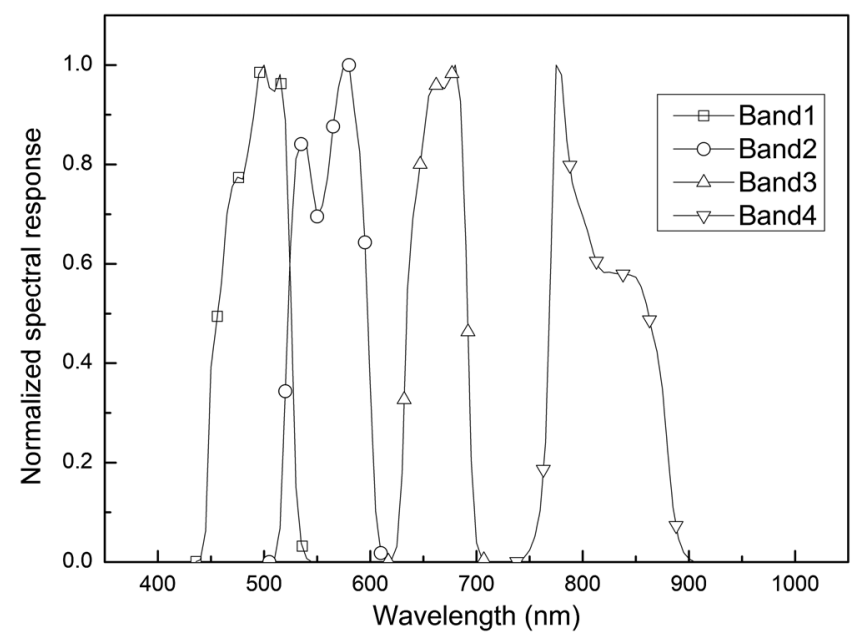

Fig. 6 SRF of the CBERS-04 WFI sensor. 


\section{Results and Discussion}

\subsection{Angular Spectrum Measurement}

The angular spectrum was measured on August 25, 2015, at the Dunhuang calibration site. To obtain the BRDF characteristics of Dunhuang site at different solar zenith and azimuth angles, four groups of measurements were taken at different solar time intervals: $7: 15$ to $7: 45,8: 15$ to $8: 45,9: 15$ to $9: 45$, and 10:15 to 10:45. The measurement data were processed using Eqs. (2) and (4) to obtain the BRF curve and the ANIF curve. Figures 7(a) and 7(b) show the surface reflectance curves and ANIF curves for different observation azimuth angles for an observation zenith angle of $30 \mathrm{deg}$ (measurement time segment 10:15 to 10:45). It can be seen from Fig. 7 that the reflectance and ANIF curves of different observation azimuth angles are obviously different under the same observation zenith angle. The BRF curves with higher and lower values concentrate on the observation azimuth angles of $150 \mathrm{deg}, 180 \mathrm{deg}, 120 \mathrm{deg}$ and $0 \mathrm{deg}, 330 \mathrm{deg}$, and $300 \mathrm{deg}$, respectively. The former observation azimuth angle is located on the direction of solar incidence, whereas the latter observation azimuth angle is located on the direction of solar forward-scattering. The ANIF factor of the CBERS-04 satellite WFI sensor in different bands can be obtained by convoluting the spectral response curve and ANIF curve using Eq. (5). Figure 8 show the ANIF factor of the CBERS-04 WFI sensor at different observation zenith and azimuth angles, for a solar zenith angle of $35.6 \mathrm{deg}$ and a solar azimuth angle of $139.9 \mathrm{deg}$.

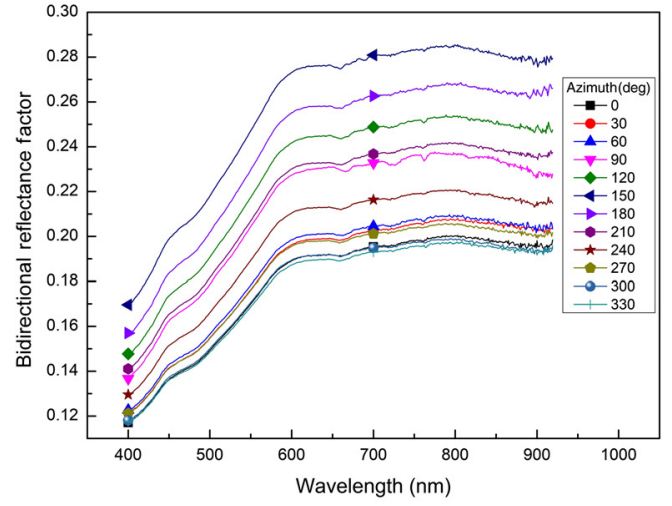

(a)

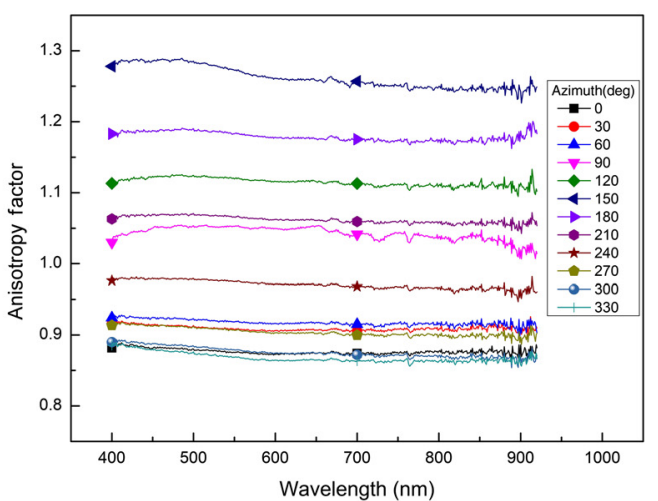

(b)

Fig. 7 (a) Surface reflectance curves and (b) ANIF curves of different observation azimuth angles for a zenith angle of 30 deg (the measurement time interval is 10:15 to 10:45).

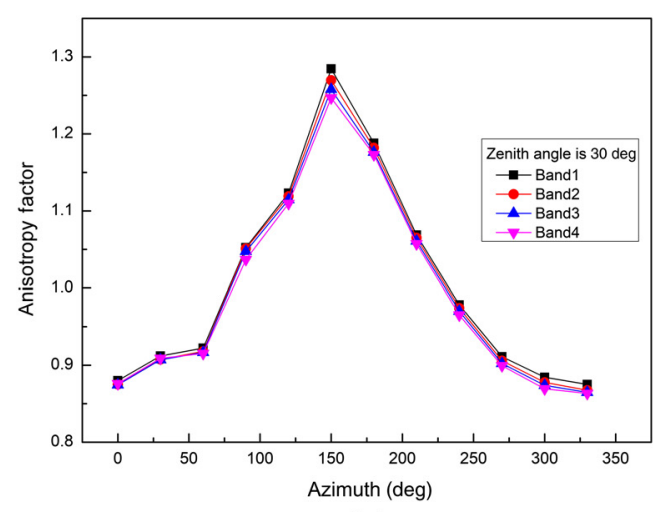

(a)

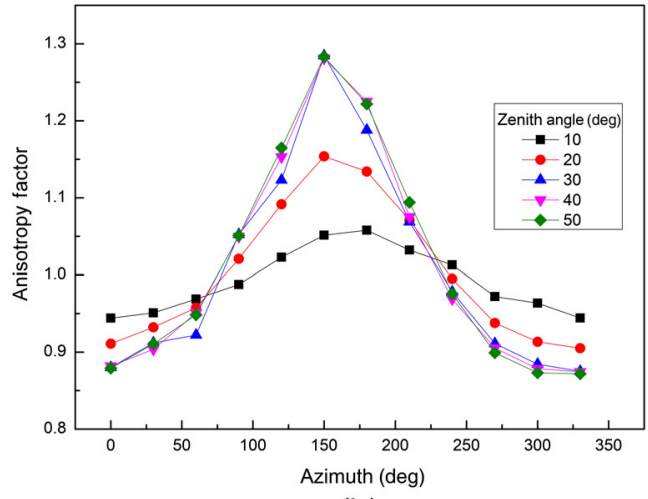

(b)

Fig. 8 ANIF factor of the CBERS-04 WFI sensor. (a) All bands at different observation azimuth angles for an observation zenith angle of $30 \mathrm{deg}$ (the measurement time segment is 10:15 to $10: 45$ ) and (b) band1 at different observation azimuth angles for observation zenith angles ranging from $10 \mathrm{deg}$ to $50 \mathrm{deg}$. 
The ANIF factors of different bands have closer values at the same observation zenith and azimuth angles. However, in the case of the same observation zenith angle, the ANIF factor of the same band varies greatly under different observation azimuth angles. The ANIF value shows a normal distribution as the azimuth ranges from 0 deg to $360 \mathrm{deg}$. For example, when the observation zenith angle is $30 \mathrm{deg}$, the maximum and minimum values of the ANIF factor for band 1 are 1.2845 and 0.8751 at observation azimuth angles of $150 \mathrm{deg}$ and $330 \mathrm{deg}$, respectively. This means that in this view angle the reflectance value is $28.45 \%$ higher or $12.49 \%$ lower than that of nadir viewing. The ANIF value has different normal distribution characteristics under different observation azimuth angles when the observation zenith angle ranges between $10 \mathrm{deg}$ and $50 \mathrm{deg}$. This indicates that the maximum and minimum values of the ANIF factor are distinctly different. Therefore, it is very important to consider the errors caused by observation angle during the satellite calibration process.

To analyze the spatial distribution characteristics of the BRDF, the ANIF factor of the CBERS-04 WFI sensor can be plotted on a polar diagram. Figure 9 is a polar diagram of the ANIF factor of the CBERS-04 WFI sensor using four groups of measurements obtained at different time segments. In the diagram, the ANIF factor is color-coded from low values (dark blue) to high values (bright red). Each ring represents a certain observation zenith angle from $0 \mathrm{deg}$ in the $\mathrm{CP}$ to $50 \mathrm{deg}$ at the edge. The observation azimuth angle is represented by the circle, which ranges from 0 deg to $360 \mathrm{deg}$. Each cross point between the observation zenith angle and the observation azimuth angle represents the ANIF factor calculated from UAV angular measurements at different observation zenith angle and azimuth angles. The values for other regions are

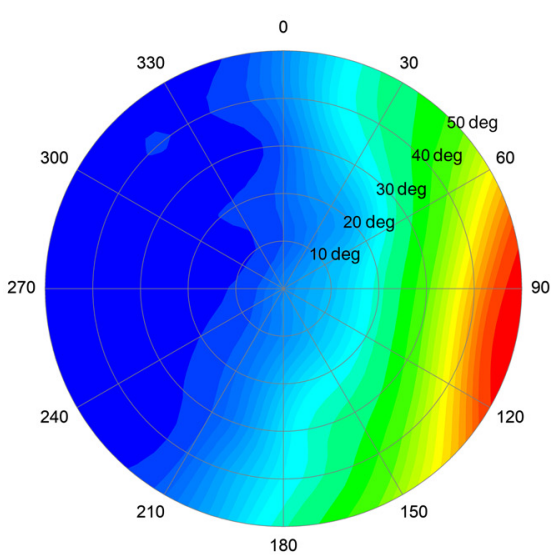

$7: 15-7: 45$

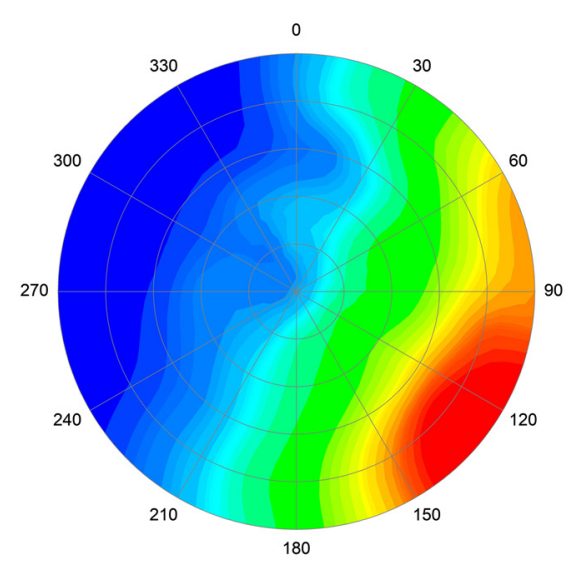

9:15-9:45
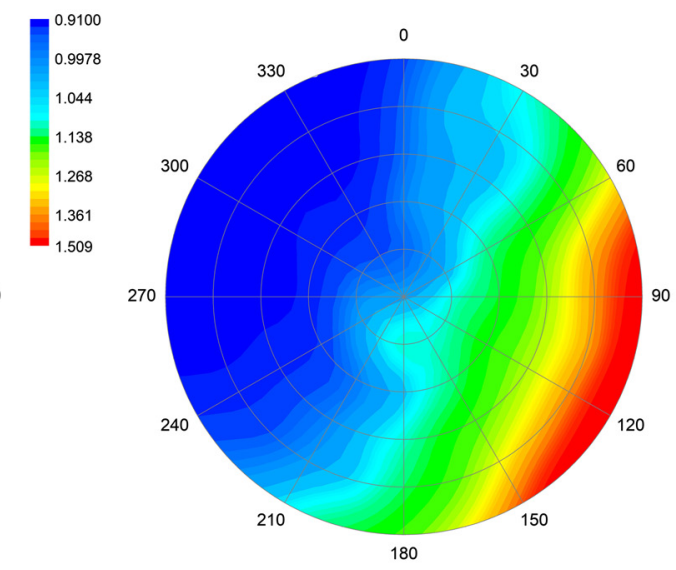

$8: 15-8: 45$
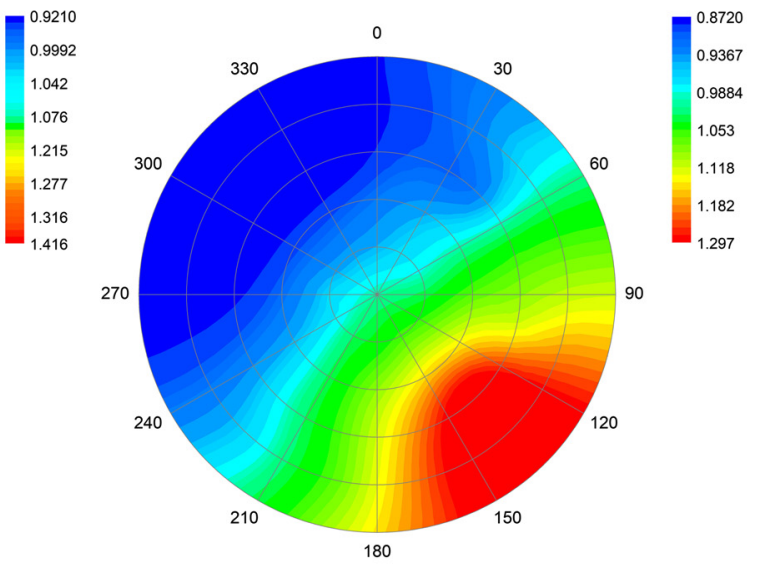

10:15-10:45

Fig. 9 Polar diagram of the ANIF factor of CBERS-04 WFI band2 measured at four different time intervals. The solar zenith angles and azimuth angles are $67.1 \mathrm{deg}, 54.2 \mathrm{deg}, 45.7 \mathrm{deg}$, and $35.6 \mathrm{deg}$ and $98.4 \mathrm{deg}, 112.8 \mathrm{deg}, 123.3 \mathrm{deg}$, and $139.9 \mathrm{deg}$, respectively. 
automatically interpolated by cross point values. From the polar diagram of the ANIF factor at different solar zenith and azimuth angles, we can make several observations: (1) there is a significant hotspot effect in each ANIF factor polar diagram, which means that reflectance is higher in the backward-scattering region where the sun is located and lower in forward-scattering regions; the surface of the Dunhuang calibration site has a backscattering effect and (2) there is different spatial distribution pattern for the ANIF factor at different solar zenith and azimuth angles. The ANIF factor diagram rotates along with the sun focal plane. The ANIF factor distribution takes the $\mathrm{CP}$ as the pivot and rotates synchronously with the sun. For a fixed observation zenith angle and azimuth angle, the ANIF factor is not constant during a day. Instead, it varies with the solar azimuth and zenith angles.

\subsection{Ross-Li Bidirectional Reflectance Distribution Function Model Construction}

To obtain directional data for any zenith and any azimuth angles of solar and observation, a BRDF model can be constructed using the measured multiangular data of the Dunhuang calibration site. The RossThick-LiSparse model is used in this study. It uses liner combinations of scattering kernels with physics calculations to simulate surface bidirectional reflectance. The RossThick-LiSparse model is expressed as Eq. (6):

$$
R\left(\theta_{s}, \theta_{v}, \phi ; \lambda\right)=f_{\text {iso }}+f_{\mathrm{vol}} K_{\mathrm{vol}}\left(\theta_{s}, \theta_{v}, \phi ; \lambda\right)+f_{\mathrm{geo}} K_{\mathrm{geo}}\left(\theta_{s}, \theta_{v}, \phi ; \lambda\right),
$$

where $R\left(\theta_{s}, \theta_{v}, \varnothing, \lambda\right)$ is the bidirectional reflectance; $\theta_{s}, \theta_{v}$, and $\varnothing$ are the solar zenith angle, view zenith angle, and relative azimuth angle, respectively; $K_{\mathrm{vol}}\left(\theta_{s}, \theta_{v}, \varnothing, \lambda\right)$ and $K_{\text {geo }}\left(\theta_{s}, \theta_{v}, \varnothing, \lambda\right)$ are the volumetric scattering kernel (RossThick kernel) and the geometric optical kernel (LiSparse kernel), both depending only on $\theta_{s}, \theta_{v}$, and $\varnothing$; and $f_{\text {iso }}, f_{\text {vol }}$, and $f_{\text {geo }}$ are the weight parameters for the isotropic, volumetric, and geometric kernels, respectively.

Taking the CBERS-04 WFI sensor as an example, the parameters of the Ross-Li BRDF model of the Dunhuang calibration field can be obtained using the measurement dataset. First, we calculate the band BRF of each measurement point using Eqs. (2) and (3). In the Eq. (6), the BRF of band $\lambda$ equals to $R\left(\theta_{s}, \theta_{v}, \varnothing, \lambda\right)$. Second, we calculate $K_{\mathrm{vol}}\left(\theta_{s}, \theta_{v}, \varnothing, \lambda\right)$ and $K_{\mathrm{geo}}\left(\theta_{s}, \theta_{v}, \varnothing, \lambda\right)$ for each measurement point. Here $K_{\mathrm{vol}}$ and $K_{\mathrm{geo}}$ are both the functions of $\theta_{s}$, $\theta_{v}, \varnothing=\left|\varnothing_{s}-\varnothing_{v}\right|$, and the calculation equations of $K_{\mathrm{vol}}$ and $K_{\mathrm{geo}}$ can be found in many literatures. ${ }^{5,8}$ The $\theta_{s}$ and $\varnothing_{s}$ can be calculated according to CP location and the measuring time of each point. The $\theta_{v}$ and $\varnothing_{v}$ of each measurement point can be obtained according to the designed flight route. Third, if the BRF, $K_{\mathrm{vol}}$ and $K_{\mathrm{geo}}$ of each measurement point are all put into Eq. (6), and the $f_{\text {iso }}, f_{\text {vol }}$, and $f_{\text {geo }}$ of the WFI sensor are obtained by the least squares method, then the BRDF model of the WFI sensor can be constructed, which can be used to calculate $R\left(\theta_{s}, \theta_{v}, \varnothing, \lambda\right)$ for any desired $\theta_{s}, \theta_{v}, \varnothing_{s}$, and $\varnothing_{v}$. A Ross-Li BRDF model program is developed for the model building and application according to AMBRALS software, which has been developed for the scientific user community by University of Massachusetts as a surrogate for the operational MODIS BRDF/Albedo code. Table 2 shows the $f_{\text {iso }}, f_{\text {vol }}$, and $f_{\text {geo }}$ of the RossThick-LiSparse model for the WFI sensor, which is constructed using the dataset of

Table 2 The $f_{\text {iso }}, f_{\text {vol }}$, and $f_{\text {geo }}$ of the RossThick-LiSparse model for the WFI sensor.

\begin{tabular}{lccc}
\hline \hline Band & $f_{\text {iso }}$ & $f_{\text {vol }}$ & $f_{\text {geo }}$ \\
\hline Band1 & 0.1936 & 0.1193 & 0.0199 \\
Band2 & 0.2323 & 0.1331 & 0.0258 \\
Band3 & 0.2566 & 0.1373 & 0.0298 \\
Band4 & 0.2656 & 0.1308 & 0.0322 \\
\hline \hline
\end{tabular}



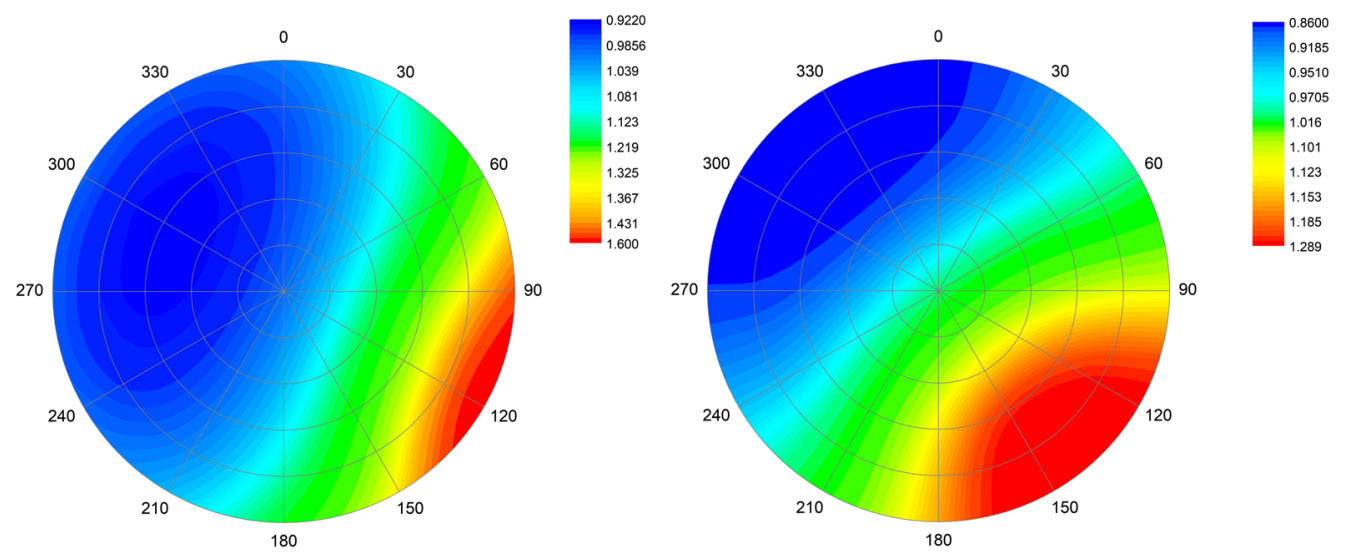

Fig. 10 Polar diagram of the ANIF factor of the CBERS-04 WFI sensor (band2), calculated using the Ross-Li model. Solar zenith angles and azimuth angles are $54.2 \mathrm{deg}, 35.6 \mathrm{deg}$ and $112.8 \mathrm{deg}$, 139.9 deg, respectively.

measurement time between 10:15 and 10:45. The measurement time is close to the imaging time of WFI sensor, and the BRDF model is used for calibration correction in Sec. 3.3.

The ANIF factor of the CBERS-04 WFI sensor (band2) was calculated using the constructed Ross-Li model when the solar zenith and azimuth angles were $54.2 \mathrm{deg}, 35.6 \mathrm{deg}$, and $112.8 \mathrm{deg}$, $139.9 \mathrm{deg}$, respectively (Fig. 10). Compared to the ANIF factors derived from field measurement data during the segments of 8:15 to 8:45 and 10:15 to 10:45 in Fig. 9, the ANIF factor calculated using Ross-Li model is consistent with those in Fig. 9 both in BRDF distribution pattern and ANIF factor value. This shows that the BRDF characteristics of the Dunhuang calibration site can be well simulated by a Ross-Li model combined with multiangular measurement data.

\subsection{BRDF Correction of WFI Sensor Calibration}

The constructed BRDF model is applied to the calibration correction of the CBERS-04 WFI sensor, which has an FOV of $\pm 32 \mathrm{deg}$. The CBERS-04 WFI sensor has passed over Dunhuang calibration site for 6 times, and the measured data include the reflectance and atmosphere data was obtained during the field calibration campaign conducted in August of 2015. Figure 11 shows the WFI images on which the Dunhuang calibration site is marked. Table 3 shows that the viewing angles of WFI sensors for the Dunhuang calibration site are very different. This should be corrected by BRDF model to obtain better calibration results.

The observed geometric parameters of the WFI sensor and solar in Table 3 are used as input to the Ross-Li model of the Dunhuang calibration site. The BRF of the WFI sensor viewing angle and WFI nadir viewing can be calculated using Eq. (6), and the ANIF factor can be obtained using Eq. (4). The ANIF factor ranges from 0.92 to 1.10 . When the calibration site is in the west part of the central line (nadir view) of the image along the track, the satellite observation azimuth angle range is between $270 \mathrm{deg}$ and $290 \mathrm{deg}$, and the ANIF factor is less than 1, meaning that the satellite observation value should be lower than the actual value of ground nadir measurement. On the contrary, when the calibration site is located on the east side of the central line (nadir view) of the image along the track, and the satellite observation azimuth angle range is between $90 \mathrm{deg}$ and $110 \mathrm{deg}$, the ANIF factor is larger than 1, meaning that the satellite observations should be higher than the actual value of the ground nadir measurement.

The ANIF factor in Table 3 is used for surface reflectance correction by multiplying the field measured reflectance (nadir) with ANIF to maintain the consistency of the viewing angle between the field measurement data and the WFI sensor. Then reflectance-based calibration method is used to calculate the calibration coefficients: the corrected/uncorrected surface reflectance, atmospheric parameters and the observed geometric parameters of solar and the satellite sensor are all input into the radiative transfer model (MODTRAN) to obtain TOA radiance, and the calibration coefficient is calculated according to the TOA radiance and DN value of the 


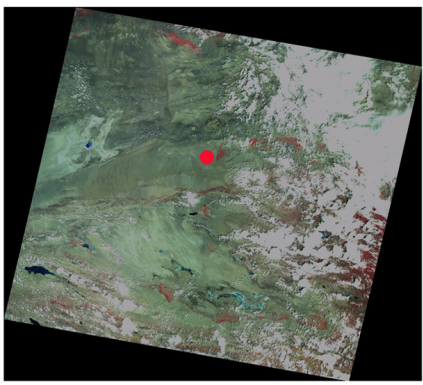

1

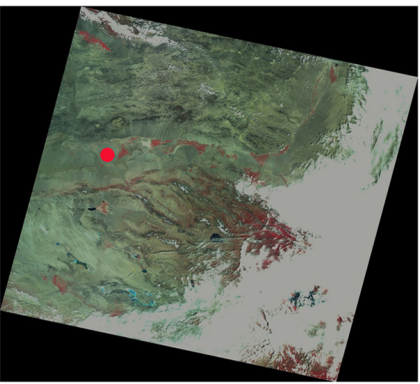

4

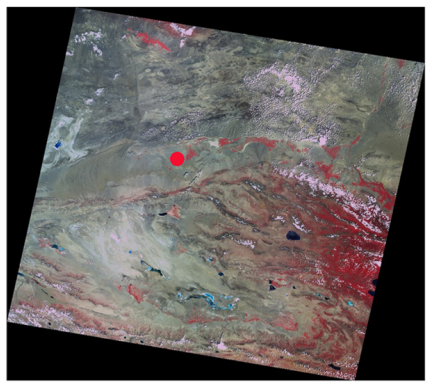

2

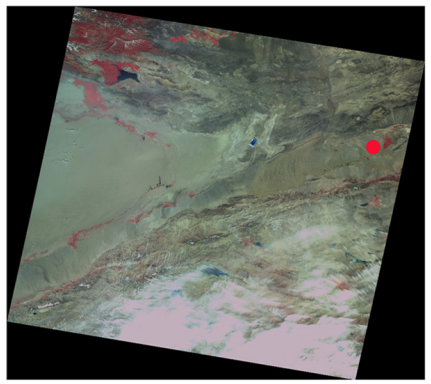

5

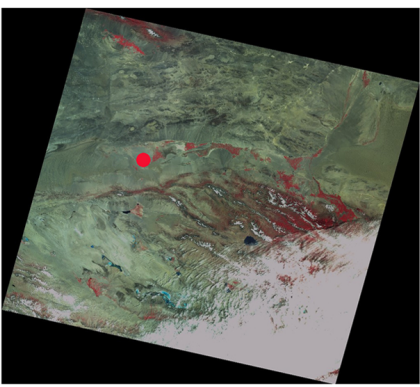

3

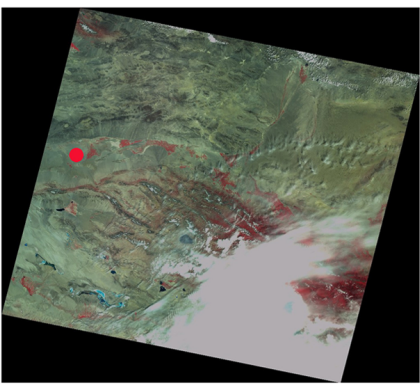

6

Fig. 11 Images of the Dunhuang calibration site taken with the WFI sensor. The location of the red dot in the image represents the position of the Dunhuang site. The acquisition dates of images 1 to 6 are listed in Table 3.

Table 3 ANIF factor at different imaging time for different bands of the WFI sensor.

\begin{tabular}{|c|c|c|c|c|c|c|c|c|c|}
\hline \multirow[b]{2}{*}{ No. } & \multirow[b]{2}{*}{ Date } & \multirow{2}{*}{$\begin{array}{l}\text { Solar } \\
\text { zenith } \\
\text { angle }\end{array}$} & \multirow{2}{*}{$\begin{array}{l}\text { Solar } \\
\text { azimuth } \\
\text { angle }\end{array}$} & \multirow{2}{*}{$\begin{array}{c}\text { Satellite } \\
\text { zenith } \\
\text { angle }\end{array}$} & \multirow{2}{*}{$\begin{array}{l}\text { Satellite } \\
\text { azimuth } \\
\text { angle }\end{array}$} & \multicolumn{4}{|c|}{ WFI ANIF } \\
\hline & & & & & & Band1 & Band2 & Band3 & Band4 \\
\hline 1 & 20150807 & 27.51 & 144.31 & 3.42 & 104.44 & 1.0128 & 1.0132 & 1.0134 & 1.0136 \\
\hline 2 & 20150810 & 28.67 & 143.54 & 9.99 & 101.42 & 1.0368 & 1.0377 & 1.0382 & 1.0389 \\
\hline 3 & 20150813 & 29.86 & 142.87 & 16.45 & 100.30 & 1.0609 & 1.0622 & 1.0627 & 1.0639 \\
\hline 4 & 20150816 & 31.07 & 142.31 & 22.75 & 99.44 & 1.0846 & 1.0862 & 1.0866 & 1.0881 \\
\hline 5 & 20150818 & 28.61 & 156.95 & 27.43 & 283.47 & 0.9285 & 0.9256 & 0.9234 & 0.9220 \\
\hline 6 & 20150819 & 32.18 & 142.23 & 27.65 & 98.80 & 1.1033 & 1.1048 & 1.1048 & 1.1067 \\
\hline
\end{tabular}

calibration site in the satellite image. Figure 12 shows the WFI calibration coefficient before and after BRDF correction. The calibration coefficients after BRDF correction of six images are linearly fitted and, for each band, one calibration coefficient is obtained, which is called as the linear fitting coefficient (LFC) (Table 4). This figure shows that the coefficients after BRDF correction are clustered more closely to the LFC than the coefficients without BRDF correction.

Table 4 shows the relative difference of WFI calibration coefficient between laboratory (A) before satellite launch and vicarious calibration. It can be found that compared to laboratory calibration coefficient the vicarious calibration coefficients after BRDF correction (C) is closer to laboratory calibration coefficient than those without BRDF correction (B). The average relative difference between (A) and (B) is $5.42 \%$, whereas the average relative difference between $(\mathrm{A})$ and $(\mathrm{C})$ is $1.74 \%$, demonstrating that the calibration accuracy is improved after BRDF correction and thus indicating the effectiveness of the constructed BRDF model. 

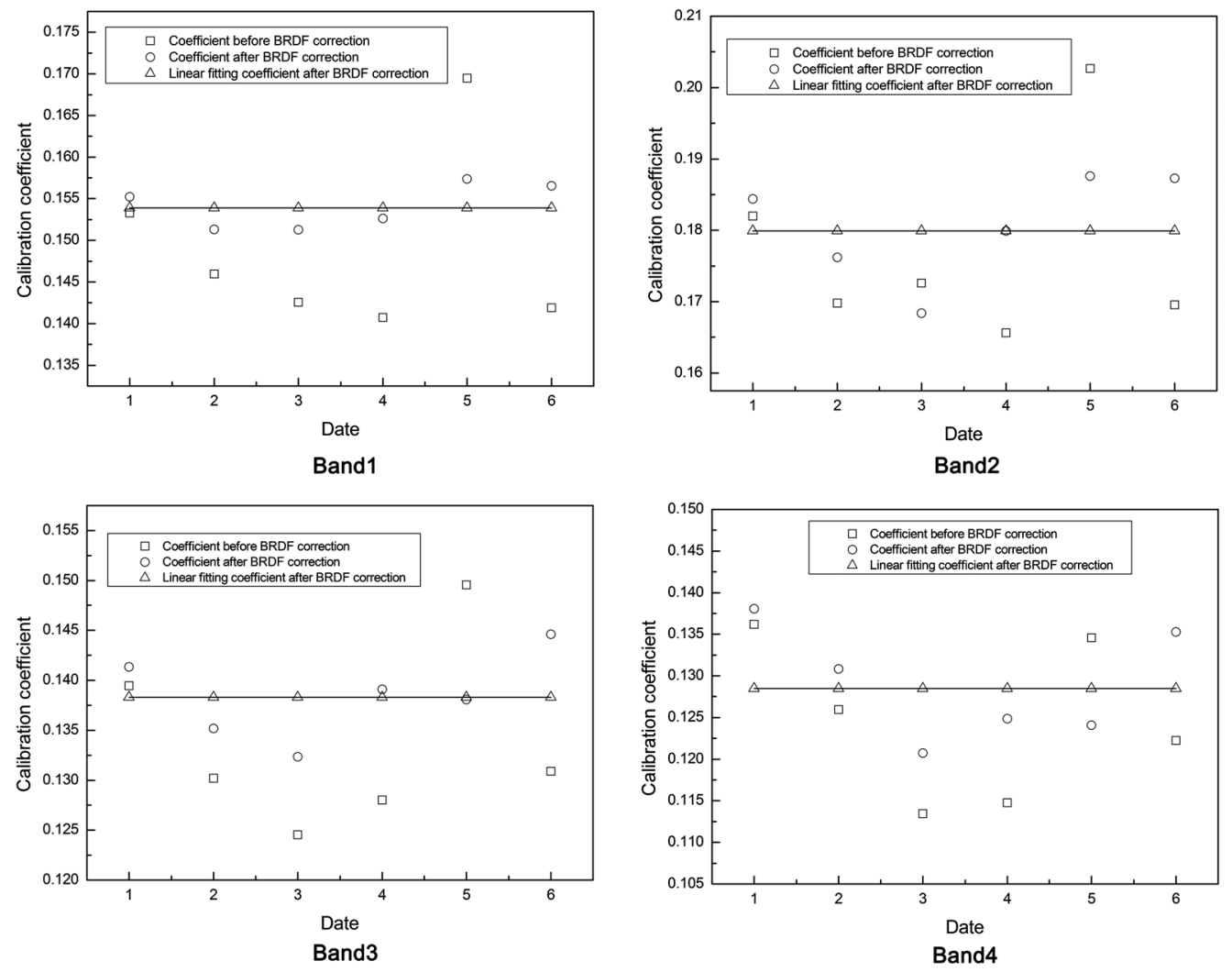

Fig. 12 WFI calibration coefficients before and after BRDF correction. The numbers on the horizontal axis correspond to the dates in Table 3.

Table 4 Relative difference of WFI calibration coefficient between laboratory and vicarious calibration before and after BRDF correction.

\begin{tabular}{lccccc}
\hline \hline & $\begin{array}{c}\text { Laboratory } \\
\text { calibration } \\
\text { coefficient (A) }\end{array}$ & $\begin{array}{c}\text { LFC before BRDF } \\
\text { correction (B) }\end{array}$ & $\begin{array}{c}\text { LFC after BRDF } \\
\text { correction (C) }\end{array}$ & $\begin{array}{c}\text { Relative } \\
\text { difference between } \\
(\mathrm{A}) \text { and (B) (\%) }\end{array}$ & $\begin{array}{c}\text { Relative } \\
\text { difference between } \\
(\mathrm{A}) \text { and (C) }(\%)\end{array}$ \\
\hline Band1 & 0.156 & 0.1476 & 0.1539 & -5.38 & -1.35 \\
Band2 & 0.179 & 0.1726 & 0.1799 & -3.58 & 0.50 \\
Band3 & 0.144 & 0.1329 & 0.1383 & -7.71 & -3.96 \\
Band4 & 0.130 & 0.1235 & 0.1285 & -5.00 & -1.15 \\
\hline \hline
\end{tabular}

Note: The unit of calibration coefficient is $\mathrm{W} \cdot \mathrm{m}^{-2} \cdot \mu \mathrm{m}^{-1} \cdot \mathrm{sr}^{-1} \cdot \mathrm{DN}^{-1}$.

\section{Conclusions}

In this study, we constructed a multiangle spectrum measurement system based on a rotor UAV. The observation azimuth angle was controlled by planning the flight route so that the heading of the UAV is aligned with the desired angle at the time of data collection. BRDF data for the Dunhuang calibration site were measured at different solar zenith angles. They were found to reflect the distribution principal of the BRDF hemisphere for a homogeneous surface such as the Dunhuang calibration site. A Ross-Li model was constructed using the measured data, and the CBERS-04 WFI sensor was calibrated using the constructed BRDF model, which improved the calibration accuracy significantly. For large FOV sensor calibration, it is very important to consider the errors caused by the inconsistent observation angle between the sensor view and the measurement view of field data. 
There is still some research work to be done. For example, the efficiency of the BRDF measurement could be improved. In this study, the observation zenith angle of the UAV measurement system was modified manually, and the landing of the UAV on the ground wasted some measurement time. In future work, the observation zenith angle should be adjusted automatically during the flight. This would allow the measurement to be completed within $15 \mathrm{~min}$. In addition, the BRDF model of the Dunhuang calibration site was constructed using measurement data collected only in the summer. Further work will be needed to verify whether the BRDF model is suitable for other seasons. In the future, additional validation of the BRDF model can be obtained by employing it to correct the results of cross calibration between two FOV sensors, such as the MODIS and WFI, in other seasons.

\section{Acknowledgments}

We would like to thank Professor Wen $\mathrm{Xu}$, who had the perspective and strategic vision to approve the China Center for Resources Satellite Data and Application (CRESDA) R\&D project that developed the unmanned aerial vehicle measurement system used for calibration. We would like to thank Professor Crystal Schaaf and Dr. Qingsong Sun from the University of Massachusetts, Boston, for providing AMBRALS software, which was very helpful in the development of the BRDF model. We would also like to thank LetPub for providing linguistic assistance during the preparation of this article. This research was funded by the National Key R\&D Program of China (Project No. 2016YFB0501504). A portion of this study was funded by a research project of CRESDA. Disclosures: The authors declare that there are no conflicts of interest in this paper.

\section{References}

1. P. N. Slater et al., "Reflectance- and radiance-based methods for the in-flight absolute calibration of multispectral sensors," Remote Sens. Environ. 22, 11-37 (1987).

2. J. S. Czapla-Myers et al., "On-orbit radiometric calibration of Earth-observing sensors using the Radiometric Calibration Test Site (RadCaTS)," Proc. SPIE 8390, 83902B (2012).

3. A. Burkart et al., "Angular dependency of hyperspectral measurements over wheat characterized by a novel UAV based goniometer," Remote Sens. 7, 725-746 (2015).

4. J. Roujean, M. Leroy, and P. Deschamps, “A bidirectional reflectance model of the earth's surface for the correction of remote sensing data," J. Geophys. Res. 97(18), 20455-20468 (1992).

5. W. Lucht and J. L. Roujean, "Considerations in the parametric modeling of BRDF and albedo from multiangular satellite sensor observations," Remote Sens. Rev. 18(2-4), 343-379 (2000).

6. X. Li and A. H. Strahler, "Geometric-optical bidirectional reflectance modeling of the discrete crown vegetation canopy: effect of crown shape and mutual shadowing," IEEE Trans. Geosci. Remote Sens. 30, 276-292 (1992).

7. W. Wanner et al., "Global retrieval of bidirectional reflectance and Albedo over land from EOS MODIS and MISR data: theory and algorithm," J. Geophys. Res. 102(14), 1714317161 (1997).

8. W. Lucht, C. B. Schaaf, and A. H. Strahler, "An algorithm for the retrieval of albedo from space using semiempirical BRDF models," IEEE Trans. Geosci. Remote Sens. 38(2), 977998 (2000).

9. C. B. Schaaf et al., "First operational BRDF, albedo nadir reflectance products from MODIS," Remote Sens. Environ. 83, 135-148 (2002).

10. P. Patel, H. Bhatt, and A. K. Shukla, "Absolute vicarious calibration of recently launched Indian meteorological satellite: INSAT-3D imager," Remote Sens. Spatial Inf. Sci. XL-8, 291-298 (2014).

11. L. Feng et al., "Radiometric cross-calibration of Gaofen-1 WFV cameras using Landsat-8 OLI images: a solution for large view angle associated problems," Remote Sens. Environ. 174, 56-68 (2016). 
12. R. Bhatt et al., "Desert based absolute calibration of visible sensors," in Geosci. Remote Sens. Symp., IEEE (2012).

13. W. Kim, S. Liang, and C. Cao, "On using BRDF models for assessment of radiometric stability of Sonoran Desert," in Geosci. Remote Sens. Symp., IEEE (2012).

14. S. Sandmeier and K. A. Itten, "Field goniometer system (FIGOS) for acquisition of hyperspectral BRDF data," IEEE Trans. Geosci. Remote Sens. 37, 978-986 (1999).

15. J. R. Jensen and S. R. Schill, "Bidirectional reflectance distribution function (BRDF) characteristics of Smooth Cordgrass (Spartina alterniflora) obtained using a Sandmeier field goniometer," Geocarto Int. 15(2), 23-30 (2000).

16. J. Schopfer et al., "The improved dual-view field goniometer system FIGOS," Sensors 8 , 5120-5140 (2008).

17. T. H. Painter, B. Paden, and J. Dozier, "Automated spectro-goniometer: a spherical robot for the field measurement of the directional reflectance of snow," Rev. Sci. Instrum. 74, 51795188 (2003).

18. C. S. Bourgeois et al., "IAC ETH goniospectrometer: a tool for hyperspectral HDRF measurements," J. Atmos. Oceanic Technol. 23, 573-584 (2006).

19. K. Z. Doctor et al., "Wavelength dependence of the bidirectional reflectance distribution function (BRDF) of beach sands," Appl. Opt. 54(31), F243-F255 (2015).

20. M. Buchhorn, R. Petereit, and B. Heim, "A manual transportable instrument platform for ground-based spectro-directional observations (mantis) and the resultant hyperspectral field goniometer system," Sensors 13, 16105-16128 (2013).

21. T. Hakala, J. Suomalainen, and J. I. Peltoniemi, "Acquisition of bidirectional reflectance factor dataset using a micro unmanned aerial vehicle and a consumer camera," Remote Sens. 2, 819-832 (2010).

22. P. Nandy, K. Thome, and S. F. Biggar, "Instrument for retrieval of BRDF data for vicarious calibration,” in Int. Geosci. and Remote Sens. Symp., Anon, Ed., Vol. 2, pp. 562-564 (1998).

23. P. Nandy, K. Thome, and S. F. Biggar, "Characterization and field use of a CCD camera system for retrieval of bidirectional reflectance distribution function," J. Geophys. Res.: Atmos. 106(D11), 11957-11966 (2001).

24. C. A. Coburn and D. R. Peddle, "A low-cost field and laboratory goniometer system for estimating hyperspectral bidirectional reflectance," Can. J. Remote Sens. 32, 244-253 (2006).

25. J. S. Czapla-Myers et al., "Directional reflectance studies in support of the Radiometric Calibration Test Site (RadCaTS) at Railroad Valley," Proc. SPIE 10764, $107640 Z$ (2018).

Zhiqiang Pan is a professor at China Centre for Resources Satellite and Application. He graduated in 2003 with a PhD from the Institute of Geographic Sciences and Natural Resources Research, Chinese Academy of Sciences, specializing in mapping science and geographic information systems. His current research interests include calibration and quantitative applications of China's land observation satellite.

Haoping Zhang is a professor of CRESDA. He received his master's degree in cartography and geographic information system. Currently, he is mainly engaged in the research of automatic radiation calibration of China's land observation satellites.

Biographies of the other authors are not available. 